\title{
Medidas generales de prevención y control contra el SARS-CoV-2: revisión de la literatura
}

\section{General measures of prevention and control of SARS-CoV-2. Literature review}

\author{
Óscar Sosa-Hernández ${ }^{1 *}$, Antonio Aguilar-Rojas ${ }^{2}$, Eduardo López-Ortiz ${ }^{3}$ \\ y Adriana MÁrQueZ-Elguea ${ }^{4}$
}

'Epidemiología, Hospital General de Zona 2, IMSS, San Luis Potosí, S.L.P.; ${ }^{2}$ Unidad de Vigilancia Epidemiológica Hospitalaria, Hospital Juárez de México, Secretaría de Salud, Ciudad de México; ${ }^{3}$ Facultad de Medicina, Subdivisión de Medicina Familiar, Universidad Nacional Autónoma de México, Ciudad de México; ${ }^{4}$ Departamento de Epidemiología, Unidad de Medicina Familiar No. 38, IMSS, San Luis Río Colorado, Son. México

\begin{abstract}
On December 31, 2019, China reported to the World Health Organization (WHO) a cluster of pneumonia cases of unknown etiology, detected in Wuhan City, Hubei Province. On February 11, 2020, the International Committee on Taxonomy of Viruses named the new coronavirus SARS-CoV-2 (severe acute respiratory syndrome coronavirus 2), and on the same day the WHO named the disease COVID-19 (coronavirus disease 2019). Here we provide an overview of this pandemic and a detailed description of the main prevention and control measures for SARSCov-2 infection.
\end{abstract}

Key words: Coronavirus. SARS-CoV-2. COVID-19. Acute respiratory infection. Emerging disease.

\section{Resumen}

El 31 de diciembre de 2019 China informó a la Organización Mundial de la Salud (OMS) sobre un conglomerado de casos de neumonía de etiología desconocida, detectados en la ciudad de Wuhan, provincia de Hubei. El 11 de febrero de 2020 el Comité Internacional sobre Taxonomía de Virus denominó al nuevo coronavirus SARS-CoV-2 (coronavirus 2 del síndrome respiratorio agudo grave) y en el mismo día la OMS denominó a la enfermedad COVID-19 (enfermedad por coronavirus 2019). El propósito de la presente revisión es brindar un panorama de lo acontecido alrededor de esta pandemia y describir de forma puntual las principales medidas de prevención y control de la infección por SARS-CoV-2.

Palabras clave: Coronavirus. SARS-CoV-2. COVID-19. Infección respiratoria aguda. Enfermedad emergente.

\section{Correspondence:}

*Óscar Sosa-Hernández

E-mail: dr.oscar.sh@gmail.com
Date of reception: 07-05-2020

Date of acceptance: 12-06-2020

DOI: 10.24875/HMCM.20000027
Available online: 09-07-2020

Hosp Med Clin Manag. 2020;13:71-6

2604-0018 / @ 2020 Mexican Regional Hospitals of High Specialty and Federal Hospitals. Published by Permanyer. This is an open access article under the CC BY-NC-ND license (http://creativecommons.org/licenses/by-nc-nd/4.0/). 


\section{INTRODUCCIÓN}

Las infecciones respiratorias agudas son la principal causa de consulta en nuestro país en todos los grupos de edad. Estos padecimientos no deberían ameritar una visita al servicio de urgencias si el paciente presenta manifestaciones leves, pero durante el invierno y la temporada de influenza este tipo de atenciones médicas saturan las instituciones de salud. A partir del mes de enero, de forma sistemática se empezó a preguntar a estos pacientes si habían estado en China recientemente o si habían tenido contacto con alguien que hubiera ido y presentara síntomas respiratorios, porque un nuevo virus fue detectado en ese país, un coronavirus del que se sabe poco pero que ya acumula más de 2.3 millones de casos y más de 479,000 defunciones en todo el mundo. El propósito de la presente revisión narrativa es brindar un panorama de lo acontecido alrededor de esta pandemia y describir de forma puntual las principales medidas de prevención y control del SARS-CoV-2 (coronavirus 2 del síndrome respiratorio agudo grave).

\section{GENERALIDADES DE LOS CORONAVIRUS}

Los coronavirus constituyen una gran familia de virus miembros de la subfamilia Orthocoronavirinae, en la familia Coronaviridae y el orden Nidovirales. Esta subfamilia consta de cuatro géneros: Alphacoronavirus, Betacoronavirus, Gammacoronavirus y Deltacoronavirus; los dos primeros infectan solo a los mamíferos y los últimos dos infectan aves principalmente, pero algunos pueden infectar también a los mamíferos ${ }^{1}$.

En los coronavirus, como en la mayoría de los virus de ácido ribonucleico (ARN), los mecanismos para reconocer y corregir mutaciones que surgen durante la replicación del genoma no son tan eficientes como en otros sistemas biológicos más complejos, como células bacterianas y animales, lo que resulta en una diversidad de especies cuyas características de adaptación a nuevos ambientes hacen posible cruzar la barrera de especies. Llegado a este punto, la afectación a las poblaciones humanas puede ser factor de tiempo ${ }^{2}$. Durante un brote por un agente infeccioso desconocido o en poblaciones sin antecedente de haber estado afectadas es importante identificar el agente etiológico por medio de la realización de toma de muestra y diagnóstico, además de definir la cadena de transmisión. El análisis de esta información es el paso inicial para diseñar intervenciones de salud pública orientadas a disminuir la magnitud del brote en contextos sociodemográficos específicos ${ }^{3}$.

\section{OTROS CORONAVIRUS DE IMPACTO MUNDIAL}

En este siglo se ha identificado la aparición y diseminación global de otros dos coronavirus desconocidos. El primero ocurrió en noviembre de 2002, cuando se detectó el primer caso del síndrome respiratorio agudo severo (SARS, por sus siglas en inglés) en Foshan, China; para febrero de 2003 ya se habían reportado más de 300 casos, el 30\% en trabajadores de la salud. En marzo de 2003 la Organización Mundial de la Salud (OMS) desplegó una red de laboratorios para establecer cuál era el agente etiológico del SARS, determinando a finales del mes de abril el SARS coronavirus (SARS-CoV) como agente causal del brote. Para el mes de julio de 2003 se habían reportado 8,096 casos, incluyendo 774 defunciones en 27 países $^{4}$. La presencia del SARS-CoV tuvo un impacto económico importante, con la pérdida mundial estimada de 40 billones de dólares, debido a gastos en bioseguridad y las restricciones impuestas al viaje y al comercio. A cambio, dejó una sociedad sensibilizada con este tipo de virus y sistemas de vigilancia epidemiológica preparados para responder ante próximas amenazas ${ }^{5}$.

En junio de 2012, en Arabia Saudita se detectó una defunción por neumonía e insuficiencia renal en un paciente de sexo masculino, en el cual se aisló un nuevo coronavirus, el coronavirus del síndrome respiratorio del Medio Oriente (MERS-CoV, por sus siglas en inglés). Este virus se caracterizó por ocasionar brotes hospitalarios: en abril de 2012 en un hospital en Jordán, en septiembre de 2012 en Reino Unido y en mayo de 2015 ocurrió uno de los brotes más grandes, en donde estuvieron involucrados 16 hospitales, con un total de 186 pacientes y 36 defunciones en Corea del Sur ${ }^{4}$. Para noviembre de 2019 se han notificado 2,494 casos confirmados de MERS-CoV, incluyendo 858 defunciones, en 27 países alrededor del mundo 6 .

\section{SITUACIÓN ACTUAL DEL BROTE DE SARS-COV-2}

El 31 de diciembre de 2019 China informó a la OMS sobre un conglomerado de casos de neumonía de 
Tabla 1. Definiciones operacionales de caso para la vigilancia epidemiológica de la enfermedad respiratoria viral

Caso sospechoso

Persona de cualquier edad que en los últimos 7 días haya presentado al menos 2 de los siguientes signos y síntomas: tos, fiebre o cefalea, acompañados de al menos uno de los siguientes signos o síntomas:

- Disnea (dato de gravedad)

- Artralgias

- Mialgias

- Odinofagia/ardor faríngeo

- Rinorrea

- Conjuntivitis

- Dolor torácico

Caso de infección respiratoria Toda persona que cumpla con la definición de caso sospechoso de enfermedad respiratoria leve y además presente aguda grave

Caso confirmado dificultad para respirar y esté hospitalizado

Persona que cumpla con la definición operacional de caso sospechoso y que cuente con diagnóstico confirmado por laboratorio de la Red Nacional de Laboratorios de Salud Pública reconocidos por el Instituto de Diagnóstico y Referencia Epidemiológicos

etiología desconocida detectados en la ciudad de Wuhan, provincia de Hubei. Del 31 de diciembre de 2019 al 3 de enero de 2020 se identificaron un total de 44 casos, todos asociados a la visita a un mercado de venta de comida de pescados y mariscos en la ciudad de Wuhan. El 7 de enero de 2020 un nuevo tipo de coronavirus fue identificado en muestras tomadas a estos pacientes, Ilamado originalmente 2019-nCoV. El 12 de enero China publicó la secuencia genética completa del virus, con el propósito de que la comunidad pudiera usarla en el diseño de estrategias preventivas (diseño de vacunas), diagnósticas (protocolos para identificación por reacción en cadena de polimerasa) y terapéuticas (desarrollo de agentes terapéuticos con objetivos moleculares específicos).

El 13 de enero Tailandia reportó el primer caso importado confirmado de 2019-nCoV, en un paciente con antecedente de viaje a la ciudad de Wuhan ${ }^{7}$. El 30 de enero la OMS declaró a este brote del nuevo coronavirus como una emergencia de salud pública de importancia internacional. El 11 de febrero de 2020 el Comité Internacional de Taxonomía de Virus nombró al nuevo coronavirus SARS-CoV-2, y en el mismo día la OMS nombró a la enfermedad COVID-19 (enfermedad por coronavirus 2019) ${ }^{8}$.

En el reporte 157 de la OMS, en el mundo se habían confirmado 9,269,202 casos y 479,133 defunciones. La región de las Américas es en la que se ha detectado el mayor número de casos, representando $49.5 \%$ de los casos confirmados y el $48.0 \%$ de las defunciones. Le sigue el continente europeo, con el $27.9 \%$ de los casos y el $40.6 \%$ de las defunciones. Sigue la región del Mediterráneo del este, con el $10.3 \%$ de los casos y el $4.5 \%$ de las. En la región del sureste asiático se tiene el 7.3\% de los casos y el 3.9\% de las defunciones; y en la región de África se han confirmado el $2.6 \%$ de los casos y el $1.1 \%$ de las defunciones ${ }^{9}$. Como en todas las enfermedades infecciosas, el panorama continuará con aumento de los casos a medida que se disemina la infección.

En México se confirmó el primer caso positivo importado el 28 de febrero; para el 23 de marzo el Consejo de Salubridad General reconoce a la COVID-19 como una enfermedad grave y da inicio la Jornada de Sana Distancia, y al mismo tiempo la OMS anuncia en su informe que en nuestro país se confirma la transmisión comunitaria $^{10}$. En cuanto al número de casos, hasta el informe del 8 de junio hubo un total de 120,102 casos confirmados, con 14,053 defunciones por COVID-1911.

\section{MEDIDAS DE PREVENCIÓN Y CONTROL EN LAS UNIDADES DE ATENCIÓN MÉDICA}

\section{Vigilancia epidemiológica}

Una de las principales actividades que se tienen que llevar a cabo para poder implementar medidas de prevención y control es la identificación pronta de los casos sospechosos y confirmados, y para este punto tenemos que revisar las definiciones operacionales de caso establecidas en nuestro país, en el Lineamiento Estandarizado para la Vigilancia Epidemiológica y por Laboratorio de la Enfermedad Respiratoria Viral, las cuales se encuentran descritas en la tabla $1^{12}$.

Al igual que con el SARS-CoV y el MERS-CoV, se ha confirmado la posibilidad de transmisión de persona a 
persona en un entorno hospitalario o de atención de la salud. Del 43.5 al $100 \%$ de los brotes de MERS-CoV fueron relacionados con los hospitales, demostrando aspectos muy parecidos a los que se presentaron en el SARS ${ }^{4}$. Las implicaciones de la transmisión de persona a persona, incluso en los asintomáticos, hacen necesario que para el control efectivo de la transmisión se fortalezca la vigilancia epidemiológica desde la capacitación del personal de primer contacto para el reconocimiento de los casos e identificación de los contactos, hasta el incremento de las capacidades diagnósticas en los laboratorios públicos, privados y académicos ${ }^{13-15}$.

\section{Lavado e higiene de manos}

Uno de los puntos críticos para romper la cadena de transmisión es el lavado e higiene de manos, por lo cual las autoridades de las unidades hospitalarias deberán garantizar la presencia de los insumos necesarios para esta actividad, es decir, agua y jabón antiséptico y soluciones antisépticas como alcohol gel. Este abasto debe ser primordial en las áreas de atención de pacientes, en las áreas de colocación y retiro de equipo de protección personal (EPP) y en el área de manejo de desechos de la atención médica como los residuos peligrosos biológico-infecciosos. Estas condiciones también deben estar disponibles para los pacientes, familiares y visitantes en las áreas de circulación general ${ }^{16}$.

Los trabajadores de la salud deben realizar el lavado e higiene de manos con la técnica adecuada establecida por la OMS, y recordar que se debe hacer en los «cinco momentos para la higiene de manos», siendo enfáticos en la colocación y retirada del EPP ${ }^{16,17}$.

\section{Equipo de protección personal}

El EPP sirve como barrera física que protege al personal de salud cuando se usa de forma adecuada. Debemos recordar que esta es una de las medidas en materia de prevención y control de infecciones; tener la disponibilidad de EPP por sí solo no es suficiente para prevenir contagios. Si no hay estricta adherencia a los procesos de colocación y retirada del EPP, y a su uso correcto, existe la posibilidad de transmisión por una autocontaminación ${ }^{17}$.

En caso de que se detecte un paciente sospechoso, tendrán que instrumentarse las medidas que se han tomado en la contención de casos de SARS, como las precauciones para la transmisión por vía de gotas y contacto, donde se debe utilizar EPP en el que se incluya cubrebocas, bata, guantes y lentes protectores; y en caso de intubar, extubar, realizar maniobras de resucitación, aspirar de forma directa o realizar alguna broncoscopia, se deberá utilizar mascarilla de alta eficiencia conocida como $\mathrm{N} 95^{18}$.

Es muy importante el uso de mascarillas N95. Hasta el momento, los protocolos establecidos indican que deben ser desechadas después del uso ante pacientes sospechosos y/o confirmados. Sin embargo, existe un problema tangible, que es el desabasto de estas mascarillas de alta eficiencia. EE.UU. calcula que necesitará 3 mil 500 millones de N95 para todos los trabajadores de la salud durante esta pandemia ${ }^{19,20}$. La OMS también publicó recientemente un comunicado donde destaca la grave escasez de EPP, lo que llevaría a un incremento en el riesgo de contagio del personal de salud $^{21,22}$.

Por esta razón, en diferentes países se están proponiendo metodologías para reutilizar las mascarillas N95. Narla, et al. hacen una breve revisión del uso de luz ultravioleta (UVC por sus siglas en inglés), donde se menciona que para utilizar esta metodología se tendría que garantizar que se logre una dosis de 1 joule por centímetro cuadrado $\left(1 \mathrm{~J} / \mathrm{cm}^{2}\right)$ para poder reutilizar esa mascarilla; sin embargo, esto puede verse afectado por la gran variabilidad de equipos de UVC y de mascarillas N95 disponibles en el mercado22.

Carrillo, et al. proponen el uso de la esterilización por vapor de uso inmediato, que fue utilizada en mascarillas N95, en las cuales la integridad en la estructura y la eficacia se mantuvieron posterior a la realización de este proceso. ${ }^{19} \mathrm{Es}$ importante mencionar que este proceso se considera tan eficaz como cualquier otra metodología de esterilización terminal ${ }^{23}$.

\section{Actividades de control de riesgo}

Dentro de un hospital idealmente se deberá mantener al paciente en una habitación individual, y tendrá que estar bajo precauciones por gotas; adicionalmente tendrán que implementarse las medidas preventivas de contacto y vía aérea debido a los diferentes mecanismos de transmisión del SARS-CoV- $2^{19}$. Idealmente tendrían que tenerse en las áreas de hospitalización y 
quirófanos filtros de alta eficiencia para partículas aéreas, se tendría que llevar a cabo la eliminación de medicamentos nebulizados, ventilación no invasiva y algunas intervenciones dentro de la organización, como contar con un equipo de respuesta rápida, establecer un triaje respiratorio, suspensión de cirugías electivas, referencia de pacientes, disminución de consulta externa y cancelación de estudios diagnósticos de pacientes externos, restricción de visitantes al hospital, entre otras que sean consideradas por las autoridades de dicha institución ${ }^{24-27}$.

\section{IMPACTO EN LA SALUD GLOBAL}

No debemos olvidar que el $60 \%$ de las enfermedades infecciosas en humanos son zoonóticas. Cinco nuevas enfermedades humanas aparecen cada año, de las cuales tres son de origen animal, y hasta el $80 \%$ de los agentes patógenos que pueden utilizarse con fines de bioterrorismo son zoonóticos ${ }^{28}$.

Un aspecto que muchas veces dejamos de lado es el aspecto económico. Las zoonosis están estrechamente asociadas con la economía, lo cual puede llevar a grandes pérdidas económicas. Por ejemplo, en el brote de Ébola, se estima que Guinea, Liberia y Sierra Leona perdieron 2.2 mil millones de dólares en producto interno bruto; otro ejemplo es el brote de influenza $A(H 5 N 2)$ en el 2014 en EE.UU., el cual resultó en una pérdida económica estimada en 3.3 mil millones de dólares. Por esta razón, y con relación a lo anteriormente descrito, invertir en medidas de prevención podría generar un ahorro de costos a medida que se evitan nuevos brotes ${ }^{29}$

\section{CONCLUSIONES}

Es importante mantenernos actualizados en temas de enfermedades emergentes y reemergentes y trabajar en conjunto todas las áreas de la salud, combinando de forma transversal las áreas de epidemiología, urgencias y de primer contacto para poder aplicar los protocolos de detección, prevención y control de infecciones de forma temprana. Debemos de tomar en cuenta que las amenazas a la salud de los coronavirus y de patógenos aún desconocidos son constantes y a largo plazo.

Comprender los procesos biológicos y sociales de estas enfermedades es fundamental para controlar su propagación, tarea en la cual todos los actores tenemos un papel que, en el caso de no ejercerlo de forma responsable y solidaria, se reflejará con implicaciones importantes para la salud global.

\section{FINANCIAMIENTO}

La presente investigación no ha recibido ayudas específicas provenientes de agencias del sector público, sector comercial o entidades sin ánimo de lucro.

\section{CONFLICTO DE INTERESES}

Los autores declaran no tener conflicto de interés alguno.

\section{RESPONSABILIDADES ÉTICAS}

Protección de personas y animales. Los autores declaran que para esta investigación no se han realizado experimentos en seres humanos ni en animales.

Confidencialidad de los datos. Los autores declaran que en este artículo no aparecen datos de pacientes.

Derecho a la privacidad y consentimiento informado. Los autores declaran que en este artículo no aparecen datos de pacientes.

\section{BIBLIOGRAFÍA}

1. Woo PC, Lau SK, Lam CS, Lau CC, Tsang AK, Lau JH, et al. Discovery of seven novel mammalian and avian coronaviruses in the genus deltacoronavirus supports bat coronaviruses as the gene source of alphacoronavirus and betacoronavirus and avian coronaviruses as the gene source of gammacoronavirus and deltacoronavirus. J Virol. 2012;86(7): 3995-4008.

2. Eckerle LD, Becker MM, Halpin RA, Li K, Venter E, Lu X, et al. Infidelity of SARS-CoV Nsp14-exonuclease mutant virus replication is revealed by complete genome sequencing. PLoS Pathog. 2010 May 6;6(5):e1000896.

3. Fehr AR, Perlman S. Coronaviruses: an overview of their replication and pathogenesis. Methods Mol Biol. 2015;1282:1-23.

4. de Wit E, van Doremalen N, Falzarano D, Munster VJ. SARS and MERS: recent insights into emerging coronaviruses. Nat Rev Microbiol. 2016; 14(8):523-34.

5. Knobler S, Mahmoud A, Lemon S, Mack A, Sivitz L, Oberholtzer K. Aprendiendo de SARS: preparándonos para el siguiente brote infeccioso. Washington DC: National Academy Press; 2004.

6. Middle East respiratory syndrome coronavirus (MERS-CoV) [Internet]; Organización Mundial de la Salud; 2019 [fecha de consulta: 20 de enero de 2020]. Disponible en: https://www.who.int/emergencies/mers-cov/en/

7. Novel Coronavirus (2019-nCoV). Situation report - 1 [Internet]. Organización Mundial de la Salud; 21 de enero de 2020 [fecha de consulta: 1 de enero de 2020]. Disponible en: https://www.who.int/docs/default-source/ coronaviruse/situation-reports/20200121-sitrep-1-2019-ncov.pdf

8. Jin Y, Yang H, Ji W, Wu W, Chen S, Zhang W, et al. Virology, epidemiology, pathogenesis, and control of COVID-19. Viruses. 2020;12:372. 
9. Coronavirus disease 2019 (COVID-19) Situation Report -157 [Internet]. Organización Mundial de la Salud; 25 de junio de 2020 [fecha de consulta: 25 de junio de 2020]. Disponible en: https://www.who.int/docs/default-source/coronaviruse/ situation-reports/20200625-covid-19-sitrep-157.pdf?sfvrsn=423f4a82 2

10. 095. Inicia fase 2 por coronavirus (COVID-19). México: Secretaría de Salud [fecha de consulta: 8 de junio de 2020]. Disponible en: https://www.gob. $\mathrm{mx} /$ salud/prensa/095-inicia-fase-2-por-coronavirus-covid-19?idiom=es

11. Coronavirus (COVID-19) - Comunicado Técnico Diario. México: Secretaría de Salud [fecha de consulta: 8 de junio de 2020]. Disponible en: https:// www.gob.mx/salud/documentos/coronavirus-covid-19-comunicado-tecnico-diario-238449

12. Lineamiento estandarizado para la vigilancia epidemiológica y por laboratorio de la enfermedad respiratoria viral [Internet]. México: Dirección General de Epidemiología, Secretaría de Salud; abril de 2020. Disponible en: https://www.gob.mx/cms/uploads/attachment/file/546206/Lineamiento estandarizado_para_la_VE_y_Lab_Enfermedad_Respiratoria_Viral.pdf

13. Kawana A, Teruya K, Kirikae T, Sekiguchi J, Kato Y, Kuroda E, et al. "Syndromic surveillance within a hospital" for the early detection of a nosocomial outbreak of acute respiratory infection. Jpn J Infect Dis. 2006;59(6):377-9.

14. Aguilar JB, Faust JS, Westafer LM, Gutierrez JB. Investigating the impact of asymptomatic carriers on COVID-19 transmission. medRxiv. 2020: 2020.03.18.20037994

15. Report 16: Role of testing in COVID-19 control [Internet]. Imperial College London, MRC Centre for Global Infectious Disease Analysis; 23 de abril de 2020. https://www.imperial.ac.uk/mrc-global-infectious-disease-analysis/ covid-19/report-16-testing/

16. Recommendation to Member States to improve hand hygiene practices widely to help prevent the transmission of the COVID-19 virus [Internet]. World Health Organization; 2020. Disponible en: https://www.who.int/docs/ default-source/inaugural-who-partners-forum/who-interim-recommendation-on-obligatory-hand-hygiene-against-transmission-of-covid-19.pdf

17. Hand Hygiene: Why, How \& When? World Health Organization; 2009. Disponible en: https://www.who.int/gpsc/5may/Hand_Hygiene_Why_How_ and_When_Brochure.pdf?ua $=1$

18. Narra, R, Sobel J, Piper C, Gould DM, Bhadelia N, Dott M, et al. CDC safety training course for ebola virus disease healthcare workers. Emerg Infect Dise. 2017;23(13).
19. Infection prevention and control during health care when COVID-19 is suspected [Internet]. World Health Organization; 2020. Disponible en: https://www.who.int/emergencies/diseases/novel-coronavirus-2019/technical-guidance/infection-prevention-and-control

20. Carrillo I, Floyd A, Valverde C, Tingle T, Zabaneh F. Immediate use steam sterilization (IUSS) sterilizes N95 masks without mask damage. Infect Control Hosp Epidemiol. 2020:17:1-5.

21. Shortage of personal protective equipment endangering health workers worldwide [Internet]. World Health Organization; 2020 [fecha de consulta: 21 de abril de 2020]. Disponible en: https://www.who.int/news-room/ detail/03-03-2020-shortage-of-personal-protective-equipment-endangering-health-workers-worldwide

22. Narla S, Lyons AB, Kohli I, Torres A, Parks-Miller A, Ozog DM, et al. The importance of the minimum dosage necessary for UVC decontamination of N95 respirators during the COVID-19 pandemic. Photodermatol Photoimmunol Photomed. 2020 Apr 14;10.1111/phpp.12562. doi: 10.1111/ phpp.12562. Online ahead of print.

23. Seavey R. Immediate use steam sterilization: Moving beyond current policy. Am J Infect Control. 2013;41(5 Suppl):S46-8.

24. Thomas PA. Severe acute respiratory syndrome. Indian J Med Microbiol. 2003;21(3):152-60

25. Siegel JD, Rhinehart E, Jackson M, Chiarello L; HICPAC. Guideline for isolation precautions: Preventing transmission of infectious agents in healthcare settings. Am J Infect Control. 2007;35(10 Suppl 2):S65-164.

26. Oh MD, Park WB, Park SW, Choe PG, Bang JH, Song KH, et al. Middle East respiratory syndrome: what we learned from the 2015 outbreak in the Republic of Korea. Korean J Intern Med. 2018;33(2):233-46.

27. Una sola salud. [Online]. Organización Mundial de Sanidad Animal; 2019 [fecha de consulta: 20 de enero de 2020]. Diesponible en: https://www.oie. int/es/para-los-periodistas/una-sola-salud/

28. Xie T, Liu W, Anderson BD, Liu X, Gray GC. A system dynamics approach to understanding the One Health concept. PLoS One. 2017:12(9):e0184430.

29. Reporte 2: Estimando el número total potencial de casos del nuevo coronavirus en la ciudad de Wuhan, China [Internet]. Imperial College London, MRC Centre for Global Infectious Disease Analysis; 2020 [fecha de consulta: enero 2020]. Disponible en: https://www.imperial.ac.uk/mrc-global-infectious-disease-analysis/news--wuhan-coronavirus/ 